\title{
Differential miRNAomics of the synovial membrane in knee osteoarthritis induced by bilateral anterior cruciate ligament transection in rats
}

\author{
JIANGTAO ZHOU ${ }^{1,2}$, YINA ZHAO $^{3}$, GUANGWEN WU $^{1}$, BINGBING LIN ${ }^{4}$, \\ ZUANFANG LI ${ }^{1,4}$ and XIANXIANG LIU ${ }^{1,5}$
}

\begin{abstract}
${ }^{1}$ Academy of Integrative Medicine, Fujian University of Traditional Chinese Medicine, Fuzhou, Fujian 350122;
Departments of ${ }^{2}$ Orthopedics and Traumatology, and ${ }^{3}$ Pharmacy, Affiliated Wenzhou Hospital

of Traditional Chinese Medicine, Zhejiang University of Traditional Chinese Medicine, Wenzhou, Zhejiang 325000;

${ }^{4}$ Fujian Key Laboratory of Rehabilitation Technology, Fujian University of Traditional Chinese Medicine,

Fuzhou, Fujian 350003; ${ }^{5}$ Fujian Provincial Key Laboratory of Integrative Medicine on Geriatrics,

Fujian University of Traditional Chinese Medicine, Fuzhou, Fujian 350122, P.R. China
\end{abstract}

Received October 25, 2017; Accepted July 13, 2018

DOI: $10.3892 / \mathrm{mmr} .2018 .9385$

\begin{abstract}
The differential microRNA (miRNA) omics of the synovial membrane were investigated using a rat model of knee osteoarthritis (KOA) induced by bilateral anterior cruciate ligament transection, which produced pathological biomarkers in KOA. Sprague-Dawley rats were randomly divided into two groups; Sham-operated and KOA-operated group. The KOA rats were subjected to bilateral anterior cruciate ligament transection. After 6 weeks, total RNA was extracted from the knee joint synovial membrane of the rats and a microRNA (miR) microarray was performed to identify differentially expressed miRs. Subsequently, the obtained differentially expressed miRs were validated by reverse transcription-quantitative polymerase chain reaction (RT-qPCR) analysis. A total of 24 miRs were identified with alterations $\geq 1.5$-fold in the synovial membrane in the KOA-operated group compared with the sham-operated group, of which 4 miRs (miR-532-5p, -200b-5p, -377-3p and -759-5p) were decreased and $20 \mathrm{miRs}(\mathrm{miR}-382-3 \mathrm{p},-223-3 \mathrm{p},-100-5 \mathrm{p}$, -30d-5p, -183-5p, -130, -92b-3p, -125b-3p, -151-3p, -155-3p, 27a-3p, -146b-3p, -885-5p, -352, -184, -345-5p, -30a-5p and $-9 \mathrm{a}-5 \mathrm{p})$ were increased. Subsequently, RT-qPCR was used to validate the expressions of miR-223, $-100,-345,-130,-382$, $-377,-352,-200 \mathrm{~b},-9 \mathrm{a}$ and -183 , which were upregulated by
\end{abstract}

Correspondence to: Professor Xianxiang Liu, Academy of Integrative Medicine, Fujian University of Traditional Chinese Medicine, 1 Qiuyang Road, Minhou Shangjie, Fuzhou, Fujian 350122, P.R. China

E-mail: liuxianxiangfz@163.com

Key words: microRNAomics, knee osteoarthritis, synovial membrane, microRNA-223, NACHT, LRR and PYD domainscontaining protein 3 inflammasome a fold change of $\geq 1.5$ in synovial membranes of KOA rats compared with shams. Furthermore, in vitro miR-223 mimic could suppress the luciferase activity of NACHT, LRR and PYD domains-containing protein 3 (NLRP3) 3' untranslated region by detecting of dual luciferase reporter vector. Additionally, the expression of NLRP3, interleukin (IL)-1 $\beta$ and IL-18 significantly increased in the synovial membrane of KOA rats. A total of 24 different miRs were determined by comparing the miRNAomics in the synovial membrane of the KOA model rats. Furthermore, the miR-233-regulated NLRP3 inflammasome was implicated in synovial membrane injury, which may be an important mechanism of KOA pathogenesis.

\section{Introduction}

Knee osteoarthritis (KOA) is a common chronic osteoarthritis pain in people aged 60 years and over, characterized by progressive degeneration of the articular cartilage and synovial membrane, which seriously affects the daily activities of $\sim 18.0 \%$ of women, and $9.6 \%$ of men worldwide $(1,2)$. The occurrence of synovitis and synovial hyperplasia is severe around the cartilage in knee joints as diagnosed by magnetic resonance imaging when the suprapatellar bursa and posterior cruciate ligament of knee is damaged (3). The causes of pathological alterations in synovitis include the release of proinflammatory cytokines and oxidative stress damage. Although a number of inflammatory factors, including tumor necrosis factor $\alpha$ (TNF- $\alpha)$, interleukin (IL)-1 $\beta$, IL-18 and NACHT, LRR and PYD domains-containing protein 3 (NLRP3), are involved in the process of synovitis, there are few reports of small molecules that progressively degenerate articular synovial membrane in KOA $(4,5)$.

Previous studies indicated that microRNAs (miRNAs/miRs), conservative endogenous non-coding RNAs of 18-25 nucleotides, are implicated in synovitis and synovial hyperplasia $(6,7)$. More evidence suggests that miRs, which are altered in the 
disease could be released into plasma, including miR-29c, -93, $-126,-184,-186,-195,-345$ and $-885-5 p$, serve an important role in degeneration of KOA (8). Articular cartilage explants stimulated with IL-1 $\beta$ demonstrated that the expression of miR-23a-3p, $-27 a-3 p$ and $-27 b-3 p$ was reduced, whereas miR-23a-3p, 24-3p, 27a-3p, 27b-3p, 29c-3p, 186-5p and -378a-5p were increased; however, only miR-23a-3p and $-27 b-3 p$ were detected in knee synovial fluid of patients with KOA (9). MiR-140 was detected in chondrocytes and knee synovial fluid of patients, and its expression is negatively correlated with the severity of KOA (10). The expression of miR-98 was reduced and caused apoptosis of cartilage cells in patients with OA; conversely, the overexpression of miR-98 could inhibit apoptosis of cartilage cells (11). A total of $20 \mathrm{miRs}$ were identified to have $>2$-fold differential expression in bone samples of patients with sclerotic OA, including miR-199a-3p, -199a-5p, -590-5p and -211-5p (12). miR-9 is activated in KOA chondrocytes; however, inhibiting miR-9 could facilitate proliferation and anti-apoptotic effect of chondrocytes by regulating nuclear factor $-\kappa \mathrm{B}(\mathrm{NF}-\kappa \mathrm{B})$ in a rat model of OA (13). Inhibition of miR-320 enhances metalloproteinase 13 (MMP-13) expression; whereas, upregulation of MMP-13, NF- $\kappa$ B and mitogen-activated protein kinase induces negative feedback mechanisms to downregulate miR-320 levels in a mouse model of chondrogenesis (14).

However, a miR expression profile in the synovial membrane has not been reported in the literature. Therefore, $\mathrm{miR}$ expression profiles were screened for molecules involved in modulating the synovial membrane in a rat model of KOA. The present study aimed to provide novel molecular mechanisms underlying KOA and novel targets for treatment.

\section{Materials and methods}

Animal ethics. A total of 18 male, 12-week-old Sprague-Dawley rats, weighing 250-300 g, were used for this study. All animals were given a standard laboratory diet with drinking water and housed in individual cages with a 12-h light-dark cycle at $23 \pm 2{ }^{\circ} \mathrm{C}$. All experimental rats and procedures were approved by the Animal Care and Usage Committee of Fujian University of Traditional Chinese Medicine (Fuzhou, China).

KOA-operated rat model. Rats were subjected to bilateral knee anterior cruciate ligament transection (ACLT) (4). Prior to ACLT, rats were sedated and anesthetized appropriately with sodium pentobarbital [0.1 ml/100 g intraperitonally (IP), $40 \mathrm{mg} / \mathrm{kg}$. The knee was shaved, then sterilized and draped in a sterile manner. A medial arthrotomy was performed. Then the patella was dislocated and the anterior cruciate ligament was isolated and transected. The ACLT was confirmed with Lachman testing by the surgeon and an observer (15). Following irrigation with sterile saline solution, the wounds were closed in layers and antiseptically treated. Rats were given appropriate postoperative care and allowed free activities in individual cages.

Animal grouping. All animals were divided into two groups according to a random number table. The KOA-operated group and the sham-operated group, each group included 9 rats according to SPSS13.0 statistics software (SPSS, Inc., Chicago, IL, USA).
Synovial membrane miRNAomics detection. After 6 weeks, the rats were sacrificed by exsanguination from the femoral artery under sodium pentobarbital anesthesia $(0.1 \mathrm{ml} / 100 \mathrm{~g}$ IP, $40 \mathrm{mg} / \mathrm{kg}$ ), knee joints were removed and synovial membranes were immediately isolated from the knee joint under aseptic conditions, then washed with saline and immediately put into liquid nitrogen. Total RNA was extracted from the synovial membrane tissues in rats using the TRIzol reagent (Thermo Fisher Scientific, Inc., Waltham, MA, USA), which recovered all RNA species. RNA quantity was determined by Nanodrop spectrophotometer (ND-1000; Nanodrop Technologies; Thermo Fisher Scientific, Inc., Wilmington, DE, USA) and RNA quality was measured by gel electrophoresis. The differential miRNAomics of synovial membrane was detected by miR microarray with three independent biological replicates in the sham group and the KOA group. Following isolation of $100 \mathrm{ng}$ total RNA, miR labeling was performed by the miRCURY ${ }^{\mathrm{TM}} \mathrm{Hy}^{\mathrm{TM}} / \mathrm{Hy}^{\mathrm{TM}}$ power labeling kit (Qiagen $\mathrm{GmbH}$, Hilden, Germany), and subsequently the Hy $3^{\mathrm{TM}}$-labeled samples were hybridized with the 7th miRCURY ${ }^{\mathrm{TM}}$ LNA Array (v.18.0; Qiagen GmbH). The hybridized slides were scanned at $635 \mathrm{~nm}$ using the Axon GenePix 4000B microarray scanner (Molecular Devices, LLC, Sunnyvale, CA, USA) then data was imported into GenePix Pro 6.0 software (Molecular Devices, LLC) for grid alignment and data extraction.

Replicated intensities of $\geq 30 \mathrm{miRs}$ were chosen to calculate normalization using the median normalization. All significant differentially expressed miRs were identified by volcano plot filtering. Hierarchical clustering was performed using the TIGR Multi Experiment Viewer software (v4.6; http://mev .tm4.org/\#/welcome). A $\geq 1.5$-fold difference in normalized levels and combination with a Welch t-test that was performed without an assumption of equal variances and false discovery rate (FDR) testing corrections were used, with a FDR of 0.05 , which was used to identify differentially expressed miRs.

Reverse transcription-quantitative polymerase chain reaction $(R T-q P C R)$. The expressions of miRs were detected by RT-qPCR. Total RNA was extracted from the synovial membrane ( $n=3$ rats per group) and then reverse transcribed at $30^{\circ} \mathrm{C}$ for $10 \mathrm{~min}$ and $42^{\circ} \mathrm{C}$ for $60 \mathrm{~min}$, followed by an incubation at $70^{\circ} \mathrm{C}$ for $15 \mathrm{~min}$ to generate cDNA using Revert Aid ${ }^{\mathrm{TM}}$ First Strand cDNA Synthesis kit (Thermo Fisher Scientific, Inc.). The reverse transcription reactions at an initial denaturation for $5 \mathrm{~min}$ at $95^{\circ} \mathrm{C}$, followed by 35 cycles of $30 \mathrm{sec}$ denaturation at $95^{\circ} \mathrm{C}$, annealing for $30 \mathrm{sec}$ at $58^{\circ} \mathrm{C}$ and extension for $30 \mathrm{sec}$ at $72^{\circ} \mathrm{C}$, the final extension step was carried out at $72^{\circ} \mathrm{C}$ for $10 \mathrm{~min}$ which were amplified with primer pair miR-223 (Forward, 5'-TGTCAGTTTGTCAAATACCCC-3' and Reverse, 5'-GCTGTCAACGATACGCTACCTA-3'), miR-100 (Forward 5'-AACCCAGATCCGAACTTGTG-3' and Reverse, 5'-GCTGTCAACGATACGCTACCTA-3'), miR-345 (Forward 5'-TGCTGACCCCTAGTCCAGTGC-3', and Reverse, 5'-GCT GTCAACGATACGCTATA-3'), miR-130 (Forward 5'-CAG TGCAATGTTAAAAGGGCAT-3', and Reverse, 5'-GCTGTC AACGATACGCTACCTA-3'), miR-382 (Forward 5'-AATCAT TCACGGACAACACTT-3', and Reverse, 5'-GCTGTCAACG ATACGCTACCTA-3'), miR-377 (Forward 5'-TGAATCACA CAAAGGCAACTTTT-3', and Reverse, 5'-GCTGTCAAC GATACGCTACCTA-3'), miR-352 (Forward 5'-AGAGTA 
GTAGGTTGCATAGTA-3', and Reverse, 5'-GCTGTCAAC GATACGCTACCTA-3'), miR-200b (Forward 5'-CATCTT ACTGGGCAGCATTGGA-3', and Reverse, 5'-GCTGTCAAC GATACGCTACCTA-3'), miR-9a (Forward 5'-TCTTTGGTT ATCTAGCTGTATGA-3', and Reverse, 5'-GCTGTCAACGAT ACGCTACCTA-3'), miR-183 (Forward 5'-TATGGCACTGGT AGAATTCACT-3', and Reverse, 5'-GCTGTCAACGATACG CTACCTA-3') and U6 (Forward 5'-CTCGCTTCGGCAGCA CA-3', and Reverse, 5'-AACGCTTCACGAATTTGCGT-3') using the Plexor ${ }^{\mathrm{TM}}$ One-Step qRT-PCR System (Promega Corporation, Madison, WI, USA) in the Bio-Rad CFX96 Detection System (Bio-Rad Laboratories, Inc., Hercules, CA, USA). The primers: The fold-change in relative miR expression was determined using the $2^{-\Delta \Delta \mathrm{Cq}}$ method and U6 small nuclear RNA as the internal control (16).

Luciferase reporter assay. The interaction of miR-223 and NLRP 3 was predicted in terms of Microcosm (www. ebi.ac.uk/errors/failure.html) and Targetscan (www .targetscan.org/vert_72/) containing computationally predicted targets for miRNAs and identify potential binding sites for a given miRNA in genomic sequences by dynamic programming alignment (17). The Luciferase reporter assay was used to determinate their interaction. The luciferase reporter vectors of psiCHECK2-NLRP3 and psiCHECK2-NLRP3-Mut (GeneCopoeia, Inc., Rockville, MD, USA) were constructed, which include the wilt type or mutated putative NLRP3 3 '-untranslated region (UTR) sequence that is targeted by miR-233. The 3'-UTR of NLRP3 primers were designed for RT-qPCR detection. NLRP3-forward 1: 5'-ccccauaaacuguUU GACUGu-3'; NLRP3-reverse 1: 5'-UCUUGUCUUGUUAAC UGACC-3'; mutant (Mut) NLRP3-forward 1: 5'-ccccauaaacug uAACUGACu-3'; MutNLRP3-reverse 1: 5'-UCUUGUCUU GUUAACUGACC-3'. Each amplified product was $\sim 1,000 \mathrm{bp}$ including putative or mutated miR-223 recognition sequence on NLRP3 3'-UTR. Clones were selected by restriction digestion with XhoI and NotI. 293T cells (ATCC, Manassas, VA, USA) cultured with DMEM (Gibco; Thermo Fisher Scientific, Inc.) supplemented with 10\% FBS (Gibco; Thermo Fisher Scientific, Inc.), 2 mM L-glutamine (Gibco; Thermo Fisher Scientific, Inc.) and 100 units $/ \mathrm{ml}$ penicillin/streptomycin (Gibco; Thermo Fisher Scientific, Inc.) were seeded at a density of $3 \times 10^{4}$ cells per well in 96-well white assay plates 1 day before cell transfection. Each reporter construct was co-transfected with $100 \mathrm{nM}$ miR-223 mimic (sequence, TGTCAGTTTGTCAAATACCCC) or $100 \mathrm{nM}$ miR-223 NC mimic (sequence, ACAGTAAACAGT TTATGGGG; GeneCopoeia, Inc., Rockville, MD, USA) in 293T cells mediating Lipofectamine ${ }^{\circledR} 2000$ (Gibco, Thermo Fisher Scientific, Inc.). After 48 h, luciferase activities were observed with the Dual-Glo luciferase assay system (Promega Corporation, Madison, WI, USA). The Renilla luciferase activity was normalized compared to the firefly luciferase activity for samples. All experiments were repeated three times for same condition.

Western blotting. The synovial membrane ( $\mathrm{n}=3$ each group) was dissected and homogenized in radio-immunoprecipitation assay lysis buffer (Nanjing Jiangcheng Bioengineering Institute, Nanjing, China), and their protein concentrations were assessed using the bicinchoninic acid protein assay (Nanjing Jiangcheng Bioengineering Institute). A total of

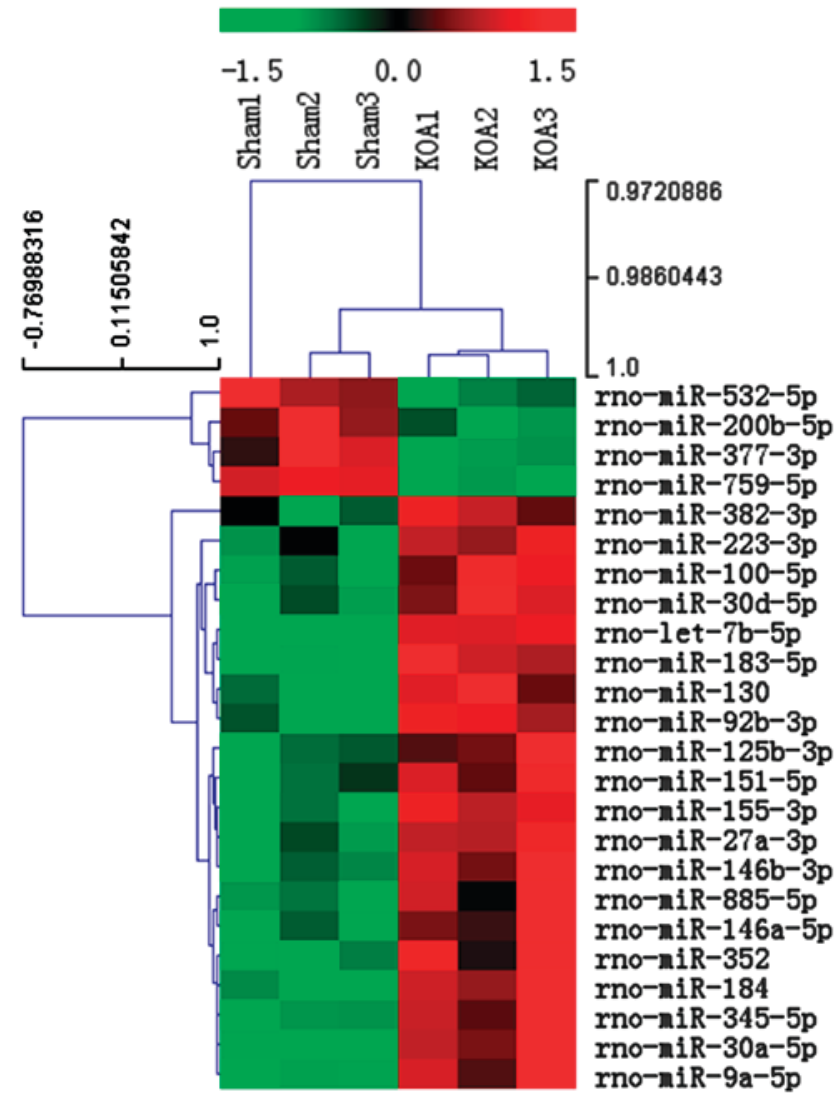

Figure 1. Identification of differentially expressed miRs in KOA rats. The differentially expressed miRs were detected in the synovial membrane of KOA rats. A total of $24 \mathrm{miRs}$ exhibited $\geq 1.5$ fold-change using cluster analysis $(\mathrm{P}<0.05$ and false discovery rate $\leq 5 \%)$. KOA group vs. sham group. Green, downregulated miRNAs; red, upregulated miRNAs. $n=3$ each group. miR, microRNA; KOA, knee osteoarthritis.

$50 \mu \mathrm{g}$ of total protein each sample was separated by $10 \%$ SDS/PAGE and transferred into nitrocellulose membrane blot (Bio-Rad Laboratories, Inc.). The blots were blocked with 3\% BSA (Life Technologies, Carlsbad, CA, USA) and incubated at room temperature $\left(22^{\circ} \mathrm{C}\right)$ for $2 \mathrm{~h}$ with primary antibodies, including rabbit monoclonal anti-NLRP3 antibody (1:500; cat. no. ab210491; Abcam, Cambridge, UK), mouse monoclonal anti-IL-1 $(1: 1,000$, cat. no. ab150777; Abcam) and IL-18 antibody (1:1,000; cat. no. ab191860; Abcam) and $\beta$-actin (1:600; cat. no. sc-47778, Santa Cruz Biotechnology, Inc., Dallas, TX, USA), and pre-adsorbed goat polyclonal secondary antibody to rabbit IgG-(1:1,000; cat. no. ab6940; Abcam) or pre-adsorbed goat polyclonal secondary antibody to mouse $\operatorname{IgG}(1: 800$; cat. no. ab97035; Abcam). Protein bands were detected with a secondary antibody conjugated to horseradish peroxidase (HRP; Jackson Immunoresearch Europe, Ltd., Newmarket, UK) and HRP was used with enhanced chemiluminescence detection (UVP, LLC, Phoenix, AZ, USA), which was quantified using a Bio-Image analysis system version 6.0.1 (Bio-Rad Laboratories, Hercules, CA, USA).

Statistical analysis. All data are expressed as the mean \pm the standard error of mean and were analyzed using SPSS 13.0 statistical analysis software (SPSS, Inc., Chicago, IL, USA). The data were subjected to a two-tailed Student's t-test analysis for comparison between two groups of the sham group and 


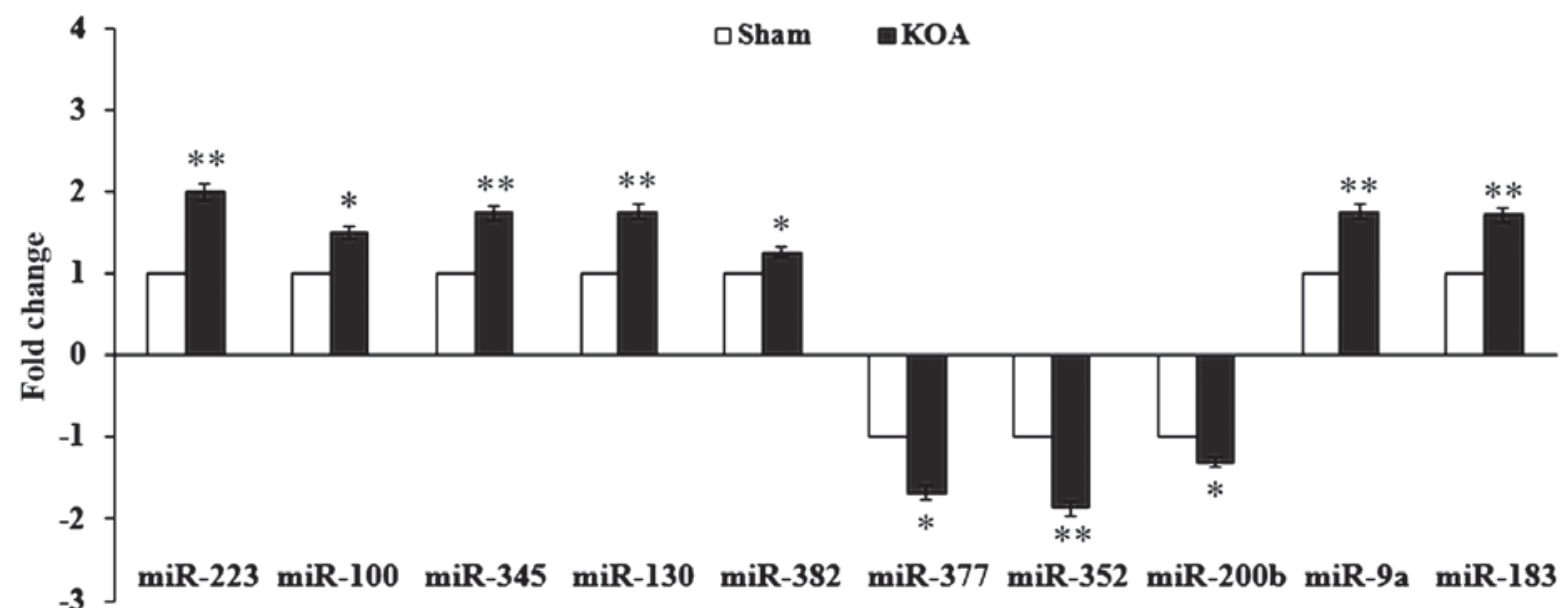

Figure 2. miRs were expressed in synovial membrane of KOA rats. The outcomes of selected 10 miRs relative expressions compared with U6 small nuclear RNA by reverse transcription-quantitative polymerase chain reaction in the synovial membrane of KOA rats ( $\mathrm{n}=3$ each group). All data are presented as the mean \pm standard error. ${ }^{*} \mathrm{P}<0.05,{ }^{* *} \mathrm{P}<0.01$ vs. Sham group. All reactions were performed in triplicate. miR, microRNA; KOA, knee osteoarthritis.

the KOA group. All final results were analyzed by observers blinded to the experimental conditions. $\mathrm{P}<0.05$ was considered to indicate a statistically significant difference.

\section{Results}

Alterations in miR expression profiling in the synovialmembrane of KOA rats. To investigate the miRs differential expression in the synovial membrane of KOA rats, miR microarray profiling was performed and a total of $24 \mathrm{miRs}$ were demonstrated to be altered by $\geq 1.5$-fold, and $\mathrm{P}<0.05$, FDR $\leq 5 \%$ in the KOA group compared with the Sham group, of which 4 miRs (miR-532-5p, $-200 b-5 p,-377-3 p$ and $-759-5 p)$ were decreased, whereas 20 miRs (miR-382-3p, -223-3p, -100-5p, -30d-5p, -183-5p, -130, -92b-3p, -125b-3p, -151-3p, -155-3p, 27a-3p, -146b-3p, -885-5p, $-352,-184,-345-5 p,-30 a-5 p$, and $-9 a-5 p)$ were increased (Fig. 1).

Expression validation of miRs by $R T-q P C R$. To confirm the $\mathrm{miR}$ expression results from the microarray, $10 \mathrm{miRs}$ were randomly chosen and assessed by RT-qPCR. The expression of miR-223, -100, -345, -130, -382, -377, -352, -200b, -9a and -183 were upregulated with a fold-change of $\geq 1.5$, similar to the microarray data (Fig. 2).

miR-233 negatively regulates the expression of NLRP3. The binding locus of the miR-223 and NLRP3 interaction was predicted in terms of Microcosm (www.ebi.ac.uk/errors/failure .html) and Targetscan (www.targetscan.org/vert_72/; Fig. 3). Subsequently, the interaction of miR-223 and NLRP3 was assessed in vitro using a luciferase reporter assay; the results suggested that the miR-223 mimic significantly inhibited the luciferase activity of NLRP3 3'UTR in 293T cells $(\mathrm{P}<0.05)$, whereas for the NLRP3 3'UTR mutant, luciferase activity was unaltered by miR-223 mimic (Fig. 3). These results suggested that miR-223 downregulated NLRP3.

Alteration of NLRP3 inflammasome-associated molecules in the synovial membrane of KOA rats. Furthermore, the expression of NLRP3 inflammasome-associated NLRP3, IL-1 $\beta$ and IL-18 proteins was measured in synovial membrane. The

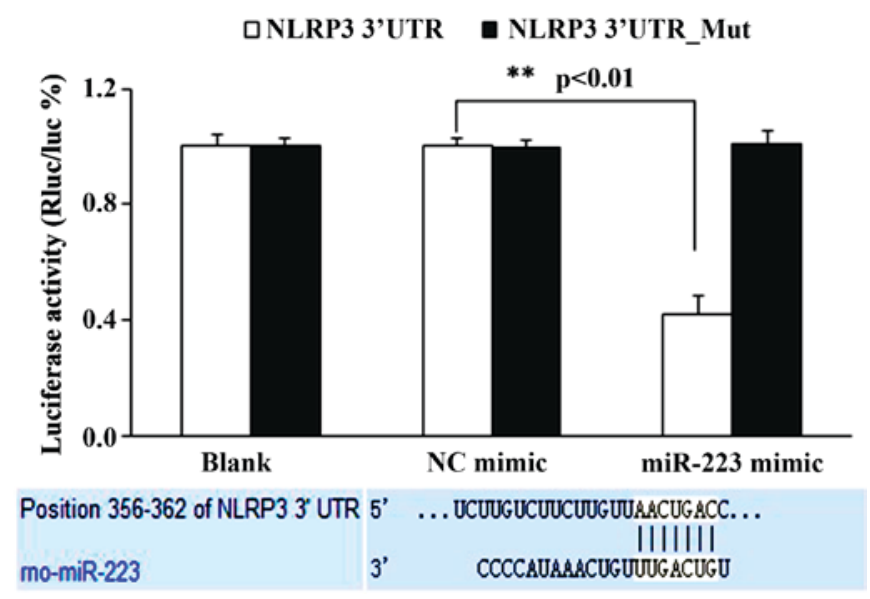

Figure 3. Identification of miR-223 and NLRP3 interaction in 293T cells. Luciferase activity of psiCHECK2-NLRP3 3'UTR and psiCHECK2-NLRP3 3'UTR mutant were measured in 293T cells transfected with miR-223 mimic ( $\mathrm{n}=4$ each group). All data are presented as the mean \pm standard error of the mean. ${ }^{* *} \mathrm{P}<0.01$. NLRP3, NACHT, LRR and PYD domains-containing protein 3; UTR, untranslated region; Mut, mutant; NC, negative control; miR, microRNA.

results demonstrated that the expression of NLRP3, IL-1 $\beta$ and IL-18 significantly increased in the synovial membrane in the KOA group compared with the Sham group $(\mathrm{P}<0.05$; Fig. 4).

\section{Discussion}

The synovial membrane is located in the joint space and maintains normal joint function by secreting hyaluronan to lubricate the tissues of joints that provide nutrition for the cartilage. However, when the synovium function is abnormal, the joint fluid is not generated and absorbed normally. The morphological alterations of the synovial membrane can also affect the cartilage of the knee joint if not treated in time, and can lead to KOA. The synovial membrane releases inflammatory factors and cytokines that result in the cartilage damage and synovial hyperplasia in patients with KOA (18). Therefore, therapeutics focus on the treatment targeting the 
A

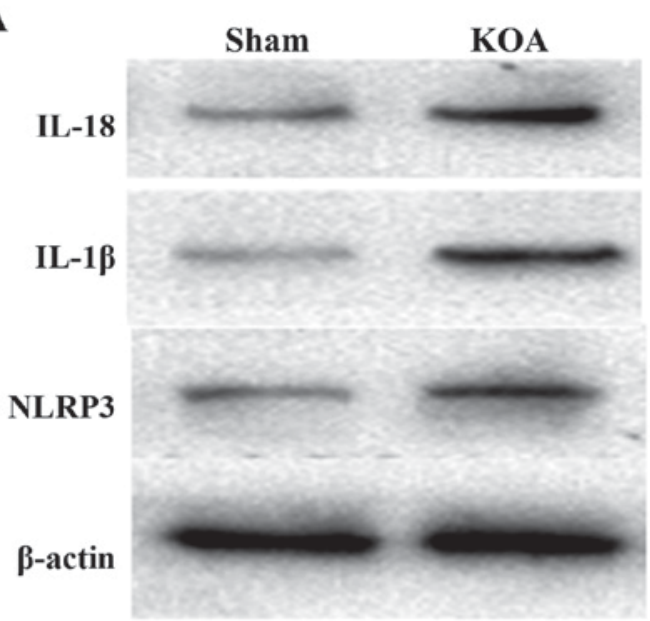

B

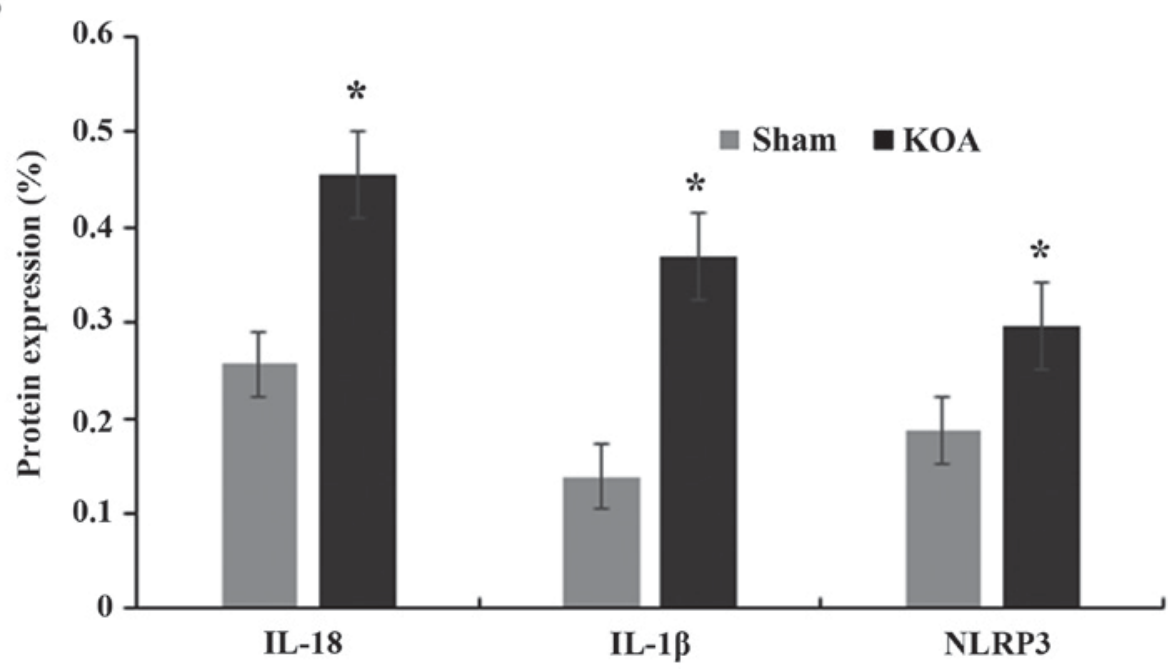

Figure 4. NLRP3, IL-1 $\beta$ and IL-18 expression in the synovial membrane of KOA rats. (A) Expression of NLRP3, IL-1 $\beta$ and IL-18 was determined in the synovial membrane of KOA rats was analyzed with western blotting ( $\mathrm{n}=3$ each group). (B) Semi-quantification of NLRP3, IL-1 $\beta$ and IL-18 levels normalized to $\beta$-actin. All data are presented as the mean \pm standard error. ${ }^{*} \mathrm{P}<0.05$ vs. sham group. KOA, knee osteoarthritis; IL, interleukin; NLRP3, NACHT, LRR and PYD domains-containing protein 3.

cartilage in KOA may be altered and that this may allow for novel treatment modalities that target the synovial membrane to be developed. In order to identify diagnostic biomarkers and therapeutic targets in different diseases, numerous studies have used miRNAomics analysis. Serum miRNAomics have been used to identify specific biomarkers of cartilage degeneration from patients with OA $(8,19)$.

However, the miRNAomics of the synovial membrane has been rarely investigated; furthermore, to the best of our knowledge, there are no studies reporting the differences in synovial membrane miRNAomics in a KOA model induced by bilateral ACLT. Therefore, the different miRNAomics of the synovial membrane were investigated via microarray in a rat model of KOA. The results illustrated the miRNAomics of the synovial membrane were different, with a total of 24 miRs exhibiting $>1.5$ fold-change in KOA rats, of which miR-532-5p, -200b-5p, -377-3p and -759-5p were downregulated, whereas miR-382-3p, -223-3p, -100-5p, -30d-5p, $-183-5 \mathrm{p},-130,-92 \mathrm{~b}-3 \mathrm{p},-125 \mathrm{~b}-3 \mathrm{p},-151-3 \mathrm{p},-155-3 \mathrm{p}, 27 \mathrm{a}-3 \mathrm{p}$, $-146 b-3 p,-885-5 p,-352,-184,-345-5 p,-30 a-5 p$ and $-9 a-5 p$ were upregulated in KOA rats compared with sham rats. Furthermore, $10 \mathrm{miR}$ were randomly selected to validate the results of miR microarrays; RT-qPCR revealed that the expression of miR-223,-100,-345,-130,-382,-377,-352,-200b, $-9 \mathrm{a}$ and -183 were downregulated by $>1.5$-fold in synovial membranes of KOA rat, which was similar to the microarray results. Using bioinformatics analysis with Microcosm and Targetscan, it was demonstrated that the rat miR-223 was implicated in inflammatory injury by interaction with the NLRP3 inflammasome. Notably, Bauernfeind et al (20) previous identified that NLRP3 inflammasome activity is negatively controlled by human miR-223. Furthermore, the miR-233 sequence is different in humans and animals: Rat, rno-miR-223-3p, 5'-UGUCAGUUUGUCAAAUACCCC-3'; human, has-miR-223-3p, 5'-TGGGGTATT TGACAA ACTGACA-3'. In addition, the locus of miR-233 binding NLRP3 in humans and rats is different. In animal experiments, it was reported that activation of Toll-like receptor 9 enhanced miR-223 expression during liver injury in vivo and in vitro (21). Nanoparticle-mediated overexpression of 
miR-223 attenuated experimental colitis, reduced NLRP3 levels and reduced IL-1 $\beta$ release (22). Elevated levels of miR-146a, miR-155 and miR-223 were demonstrated in paraffin-embedded synovial tissue of patients with established rheumatoid arthritis (23). In addition, high levels of miR-223 were present in the peripheral blood of patients with OA (24). The relative expression levels of miR-223 in patients with $\mathrm{OA}$ were reported to be significantly increased compared with those demonstrated in healthy controls; and in the early stages of OA, miR-223 expression was significantly increased compared with later stages (24).

NLRP3 is member of the NLR family characterized by binding with ribonucleotide-phosphates, which are important for self-oligomerization and are able to assemble and oligomerize into a common structure that collectively activates the caspase- 1 cascade; thus leading to the production of pro-inflammatory cytokines, particularly IL-1 $\beta$ and IL-18 $(25,26)$. Therefore, NALP3 activation leads to an inflammatory state in the osteoarthritic joint (27). In KOA models, the synovia promote the production of proinflammatory mediator that are released into the cartilage via the synovial fluid, where they activate the chondrocytes to produce more proinflammatory cytokines, including IL-1 (28). A previous study has demonstrated that IL- $1 \beta$ knockout mice were protected from surgically-induced instability OA damage (29); whereas, similar models also demonstrated that cartilage injury was exacerbated in caspase 1 and IL-1 $\beta$ knockout mice (30). Furthermore, IP injection of IL-1 in osteoarthritic mice did not relieve OA features. Additionally, the IL-1 $\beta$ level in the synovial membrane was positively correlated with OA grade, and joint space width was negatively correlated with joint activity $(31,32)$. Additionally, IL-1 $\beta$ was detected in human OA cartilage, especially in early stage OA and the IL-1 $\beta$ level in the synovial fluid was correlated with synovial fluid uric acid level in patients with KOA; thus, it was concluded that synovial fluid uric acid could be a danger signal that contributes to increasing KOA risk through NLRP3-mediated inflammasome $(33,34)$.

In conclusion, a total of 24 differentially expressed miRs were identified by comparing the miRNAomics in the synovial membrane of the KOA model and sham rats. Furthermore, the miR-233 downregulated-NLRP3 inflammasome was implicated in synovial membrane injury, which may be involved in the pathogenesis of KOA. These results revealed that miR-233 regulated NLRP3 in synovial membrane injury. Therefore, in future studies miR-233 transgenic models or miR-233 mimic and inhibitors will be used to elucidate the mechanism of miR-233 regulating targets in pathogenesis of KOA.

\section{Acknowledgements}

Authors would like to thank Dr Liu Weilin, from the Department of Rehabilitation Medicine, Fujian University of Traditional Chinese Medicine, for his helpful discussion.

\section{Funding}

The present study was supported by the National Natural Science Foundation of China (grant no. 81774345).

\section{Availability of data and materials}

The analyzed data sets generated during the study are available from the corresponding author on reasonable request.

\section{Authors' contributions}

XL and JZ designed experiments. JZ, YZ, GW, BL and ZL performed the experiments. JZ wrote the manuscript. All authors discussed the results and approved the final manuscript.

\section{Ethics approval and consent to participate}

All experimental rats and procedures were approved by Animal Care and Usage Committee of Fujian University of Traditional Chinese Medicine (Fuzhou, China).

\section{Patient consent for publication}

Not applicable.

\section{Competing interests}

The authors declare they have no competing interests.

\section{References}

1. Goldring MB and Goldring SR: Osteoarthritis. J Cell Physiol 213: 626-634, 2007.

2. Krasnokutsky S, Attur M, Palmer G, Samuels J and Abramson SB: Current concepts in the pathogenesis of osteoarthritis. Osteoarthritis Cartilage 16 (Suppl 3): S1-S3, 2008.

3. Hayashi D, Roemer FW, Katur A, Felson DT, Yang SO, Alomran F and Guermazi A: Imaging of synovitis in osteoarthritis: Current status and outlook. Semin Arthritis Rheum 41: 116-130, 2011.

4. Liu YX, Wang GD, Wang X, Zhang YL and Zhang TL: Effects of TLR-2/NF- $\kappa \mathrm{B}$ signaling pathway on the occurrence of degenerative knee osteoarthritis: An in vivo and in vitro study. Oncotarget 8: 38602-38617, 2017.

5. Berenbaum F, Eymard F and Houard X: Osteoarthritis, inflammation and obesity. Curr Opin Rheumatol 25: 114-118, 2013.

6. Ambros V: The functions of animal microRNAs. Nature 431: 350-355, 2004.

7. Wu W, He A, Wen Y, Xiao X, Hao J, Zhang F and Guo X: Comparison of microRNA expression profiles of Kashin-Beck disease, osteoarthritis and rheumatoid arthritis. Sci Rep 7: 540, 2017.

8. Borgonio Cuadra VM, González-Huerta NC, Romero-Córdoba S, Hidalgo-Miranda A and Miranda-Duarte A: Altered expression of circulating microRNA in plasma of patients with primary osteoarthritis and in silico analysis of their pathways. PLoS One 9: e97690, 2014.

9. Li YH, Tavallaee G, Tokar T, Nakamura A, Sundararajan K, Weston A, Sharma A, Mahomed NN, Gandhi R, Jurisica I and Kapoor M: Identification of synovial fluid microRNA signature in knee osteoarthritis: Differentiating early- and late-stage knee osteoarthritis. Osteoarthritis Cartilage 24: 1577-1586, 2016.

10. Si H,Zeng Y, Zhou Z, Pei F, Lu Y, Cheng J and Shen B: Expression of miRNA-140 in chondrocytes and synovial fluid of knee joints in patients with osteoarthritis. Chin Med Sci J 31: 207-212, 2016.

11. Wang GL, Wu YB, Liu JT and Li CY: Upregulation of miR-98 inhibits apoptosis in cartilage cells in osteoarthritis. Genet Test Mol Biomarkers 20: 645-653, 2016.

12. Prasadam I, Batra J, Perry S, Gu W, Crawford R and Xiao Y: Systematic identification, characterization and target gene analysis of microRNAs involved in osteoarthritis subchondral bone pathogenesis. Calcif Tissue Int 99: 43-55, 2016.

13. Gu R, Liu N, Luo S, Huang W, Zha Z and Yang J: MicroRNA-9 regulates the development of knee osteoarthritis through the NF-kappaB1 pathway in chondrocytes. Medicine (Baltimore) 95: e4315, 2016. 
14. Meng F, Zhang Z, Chen W, Huang G, He A, Hou C, Long Y, Yang Z, Zhang Z and Liao W: MicroRNA-320 regulates matrix metalloproteinase-13 expression in chondrogenesis and interleukin-1 $\beta$-induced chondrocyte responses. Osteoarthritis Cartilage 24: 932-941, 2016.

15. Fu SC, Cheng WH, Cheuk YC, Mok TY, Rolf CG, Yung SH and Chan KM: Effect of graft tensioning on mechanical restoration in a rat model of anterior cruciate ligament reconstruction using free tendon graft. Knee Surg Sports Traumatol Arthrosc 21: 1226-1233, 2013

16. Livak KJ and Schmittgen TD: Analysis of relative gene expression data using real-time quantitative PCR and the 2(-Delta Delta C(T)) method. Methods 25: 402-408, 2001.

17. Lewis BP, Shih IH, Jones-Rhoades MW, Bartel DP and Burge CB: Prediction of mammalian microRNA targets. Cell 115: 787-798, 2003.

18. Mathiessen A and Conaghan PG: Synovitis in osteoarthritis: Current understanding with therapeutic implications. Arthritis Res Ther 19: 18, 2017.

19. Kung LH, Zaki S, Ravi V, Rowley L, Smith MM, Bell KM, Bateman JF and Little CB: Utility of circulating serum miRNAs as biomarkers of early cartilage degeneration in animal models of post-traumatic osteoarthritis and inflammatory arthritis. Osteoarthritis Cartilage 25: 426-434, 2017.

20. Bauernfeind F, Rieger A, Schildberg FA, Knolle PA, Schmid-Burgk JL and Hornung V: NLRP3 inflammasome activity is negatively controlled by miR-223. J Immunol 189: 4175-4181, 2012

21. He Y, Feng D, Li M, Gao Y, Ramirez T, Cao H, Kim SJ, Yang Y, Cai Y, Ju C, et al: Hepatic mitochondrial DNA/Toll-like receptor 9/MicroRNA-223 forms a negative feedback loop to limit neutrophil overactivation and acetaminophen hepatotoxicity in mice. Hepatology 66: 220-234, 2017.

22. Neudecker V, Haneklaus M, Jensen O, Khailova L, Masterson JC, Tye H, Biette K, Jedlicka P, Brodsky KS, Gerich ME, et al Myeloid-derived miR-223 regulates intestinal inflammation via repression of the NLRP3 inflammasome. J Exp Med 214: $1737-1752,2017$.

23. Kriegsmann M, Randau TM, Gravius S, Lisenko K, Altmann C, Arens N and Kriegsmann J: Expression of miR-146a, miR-155, and miR-223 in formalin-fixed paraffin-embedded synovial tissues of patients with rheumatoid arthritis and osteoarthritis. Virchows Arch 469: 93-100, 2016.

24. Okuhara A, Nakasa T, Shibuya H, Niimoto T, Adachi N, Deie M and Ochi M: Changes in microRNA expression in peripheral mononuclear cells according to the progression of osteoarthritis. Mod Rheumatol 22: 446-457, 2012.
25. Elliott EI and Sutterwala FS: Initiation and perpetuation of NLRP3 inflammasome activation and assembly. Immunol Rev 265: 35-52, 2015.

26. Chen M, Wang H, Chen W and Meng G: Regulation of adaptive immunity by the NLRP3 inflammasome. Int Immunopharmacol 11: 549-554, 2011.

27. Clavijo-Cornejo D, Martínez-Flores K, Silva-Luna K, Martínez-Nava GA, Fernández-Torres J, Zamudio-Cuevas Y, Guadalupe Santamaría-Olmedo M, Granados-Montiel J, Pineda C and López-Reyes A: The overexpression of NALP3 inflammasome in knee osteoarthritis is associated with synovial membrane prolidase and NADPH oxidase 2. Oxid Med Cell Longev 2016: 1472567, 2016.

28. Nasi S, Ea HK, So A and Busso N: Revisiting the role of interleukin-1 pathway in osteoarthritis: Interleukin-1 $\alpha$ and $-1 \beta$, and NLRP3 inflammasome are not involved in the pathological features of the murine menisectomy model of osteoarthritis. Front Pharmacol 8: 282, 2017.

29. Ea HK, Chobaz V, Nguyen C, Nasi S, van Lent P, Daudon M, Dessombz A, Bazin D, McCarthy G, Jolles-Haeberli B, et al: Pathogenic role of basic calcium phosphate crystals in destructive arthropathies. PLoS One 8: e57352, 2013.

30. van Dalen SC, Blom AB, Slöetjes AW, Helsen MM, Roth J, Vogl T, van de Loo FA, Koenders MI, van der Kraan PM, van den Berg WB, et al: Interleukin-1 is not involved in synovial inflammation and cartilage destruction in collagenase-induced osteoarthritis. Osteoarthritis Cartilage 25: 385-396, 2017.

31. Jin C, Frayssinet P, Pelker R, Cwirka D, Hu B, Vignery A, Eisenbarth SC and Flavell RA: NLRP3 inflammasome plays a critical role in the pathogenesis of hydroxyapatite-associated arthropathy. Proc Natl Acad Sci USA 108: 14867-14872, 2011.

32. Furman BD, Mangiapani DS, Zeitler E, Bailey KN, Horne PH, Huebner JL, Kraus VB, Guilak F and Olson SA: Targeting pro-inflammatory cytokines following joint injury: Acute intra-articular inhibition of interleukin-1 following knee injury prevents post-traumatic arthritis. Arthritis Res Ther 16: R134, 2014.

33. Kolly L, Karababa M, Joosten LA, Narayan S, Salvi R, Pétrilli V, Tschopp J, van den Berg WB, So AK and Busso N: Inflammatory role of ASC in antigen-induced arthritis is independent of caspase-1, NALP-3, and IPAF. J Immunol 183: 4003-4012, 2009.

34. Scanu A, Oliviero F, Gruaz L, Galozzi P, Luisetto R, Ramonda R, Burger D and Punzi L: Synovial fluid proteins are required for the induction of interleukin-1 $\beta$ production by monosodium urate crystals. Scand J Rheumatol 45: 384-393, 2016. 